\title{
Targeting the FGFR curbs bone metastasis
}

"Growth factor receptors are overexpressed during prostate cancer progression, but whether these receptors are involved in bone metastasis is controversial," says Nora Navone from MD Anderson Cancer Center, Houston, USA. "Our studies indicate that targeting the fibroblast growth factor (FGF) receptor (FGFR) in the bone microenvironment has therapeutic activity in patients with advanced-stage prostate cancer and provides direction for therapy development using FGFR inhibitors.”

\section{4 ...targeting the [FGFR] ... in the bone microenvironment has therapeutic activity... 77}

Bone metastases are often a sign of castration-resistant prostate cancer (CRPC) and are the primary cause of morbidity and mortality in affected patients. After identifying that FGF signalling is implicated in the crosstalk of tumour cells and bone stromal cells, Navone and her team of researchers investigated the efficacy of dovitinib-an inhibitor of the FGFR and the vascular endothelial growth factor receptor -in this setting. "A 'co-clinical' and transdisciplinary approach and integration of results from mouse models and patients provided insights into FGF-FGFR biology and the mechanisms underlying the clinical activity of dovitinib that would not have been evident from preclinical models or clinical trials alone," explains Navone.

Specifically, the investigators found that mouse femurs xenografted with prostate cancer had increased Fgfrl and Fgf2 expression in comparison with contralateral control femurs. In addition, 183 human prostate tissues (cell line, xenograft and clinical samples) had increased FGFR1 expression compared with other FGFR family genes, highlighting the central role of this isoform in bone-tumour crosstalk. Next, the researchers treated mice harbouring intrafemoral xenograftsderived from subcutaneously maintained cells that had been collected from a patient with prostate cancer and had the highest FGFR1 expression-with dovitinib and found a significant reduction in tumour volumes after 3 weeks. Mechanistic studies demonstrated the activation of apoptotic pathways in the centre of tumours, indicating that dovitinib efficacy might be partly attributable to modulation of the tumour microenvironment-for example through antiangiogenic activity of the inhibitor. Notably, dovitinib antitumour activity was dependent on FGFR1 expression in xenograft tumour cells.

In a proof-of-principle study of dovitinib in humans, 34 patients with CRPC and bone metastases have been enrolled to date. Of 23 patients who have completed at least one cycle of treatment, one patient had a complete response and five patients had partial

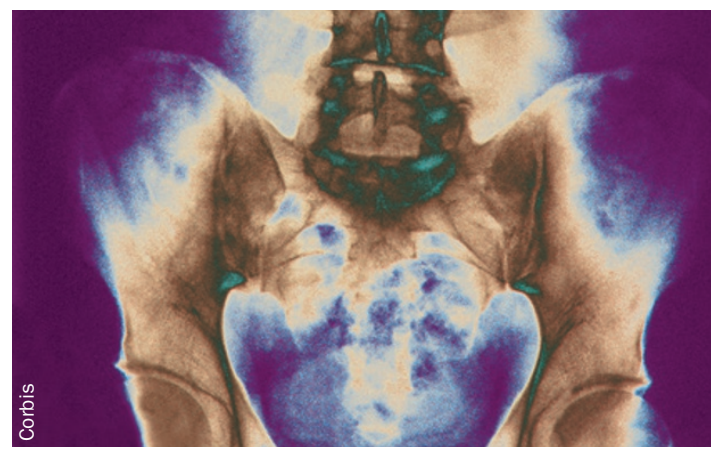

responses demonstrated by improvements in bone scans or soft-tissue metastases. Interestingly, a decrease in levels of the serum biomarker bone-specific alkaline phosphatase correlated with longer median treatment duration, indicating a correlation with treatment efficacy.

"Therapy development requires determination of rational treatment combinations and identification of markers to accurately select likely responders and evaluate treatment responses," remarks Navone. For example, a strategy targeting the stroma and epithelium has the potential to prolong lives of selected patients with CRPC and bone metastases. "To this end, a new clinical study of dovitinib plus abiraterone (an androgen biosynthesis inhibitor) in these patients is now open at our institution."

Clemens Thoma

Original article Wan, X. et al. Prostate cancer cell-stromal cell crosstalk via FGFR1 mediates antitumor activity of dovitinib in bone metastases. Sci. Transl. Med. 6, 252ra122 (2014) 